\title{
It's Never Too Early to Cleanse the Oral Cavity of Our Babies
}

\author{
Marisa Roncati and Giancarlo Couch* \\ Private Practitioner, General Dentistry, Italy \\ *Corresponding author: Giancarlo Couch, Private Practitioner, General Dentistry, Italy
}

\section{Editorial}

Dental caries continues to be the single most common chronic disease of infancy and one most widespread pathologies in the world. In Italy recent studies showed that $22 \%$ of 4 -year olds and $44 \%$ of 14 -year-olds suffer from dental caries. In industrialized nations this trend appears to be decreasing decreasing in the 6-19-year old age group. Dental caries is increasing by $15.2 \%$, According to recent epidemiological studies (2007), in the city of Milan, located in the Lombard Region of northeastern Italy and the epicenter of the Covid-19 outbreak, dental caries in children aged 2-5 years has increased by $15.2 \%$. Why are deciduous teeth more prone to caries? In part because although the enamel of deciduous teeth takes more time to develop, it demineralizes faster. Parent responsibility is also an important factor. Many parents don't begin cleaning their children's teeth until they reach 2-3 years of age and often it's not performed properly. A diet rich in sugar, snacks, candy and chocolate also plays a key role. Another cariogenic feature is the prolonged use of the baby bottle filled with sugary or acidic liquids given to the child in the evening or during the night, when there's a reduction in saliva flow.

For caries to form three factors must be present:

a) A tooth that easily demineralizes

b) Sugar in the diet

c) Specific bacteria

The first factor cannot be modified, while the other two are adjustable. Intervening on either one of these two aspects can prevent caries from forming. The easiest to correct, aimed at reducing the number of harmful bacteria, is oral hygiene. Bacteria begin developing in the infant's oral cavity following birth. At birth the oral cavity is sterile. In the children's saliva mainly bacterial strains of the respective mothers are found. The main source of infection is, therefore, maternal saliva. Primary prevention for the
2-5 age group can and must be initiated by the mother, followed by the father, the grandparents or other care givers. It's never too early to begin cleaning the child's or even the newborns oral mucosa. How? By using a clean or sterile gauze. It's is a very simple but efficient means of removing bacteria from the oral mucosa. Place the index finger in the center of an open gauze then wrap it around the index finger of your dominant hand. To soften the gauze dampen it with a normal saline solution or plain tap water. The mouth is gently opened and the oral mucosa along with the gums are delicately rubbed. This simple procedure is effective in reducing the number of bacteria, while at the same time being non traumatic to the infant or small child.

Everyone agrees that prevention is more important than treatment, but only few reward acts of prevention.

An article entitled "Oral Diseases Affect Some 3.9 Billion People", published in 2015 in the evidence-based

literature search site (Pub Med) revealed how widespread oral disease is on a global level. Today our understanding of the ferocity and aggressiveness of bacteria and viruses that inhabit or transit via the oral cavity is greater than ever before. The need to implement procedures for their mechanical reduction is crucial and it must begin at infancy. In Italy, during the period of forced lock-down due to the Covid-19 pandemic, parents became teachers, playmates, and hairdressers for their children. They invented new forms of creative entertainment even in their children's home care and "Digital Brushing" was cleverly introduced. Children would mimic their parent's gestures by rubbing their oral tissues and teeth with a gauze wrapped around their finger soaked in normal saline solution or plain water. For the child this was a game, but from an oral health standpoint this is extremely important not only in preventing disease, but in forming good, lifelong habits. A scrubbing action is indispensable to detach the adhesive biofilm from oral structures. Rinsing will not remove it. Rinsing only removes loose, 
floating debris and does not reach bacteria and viruses enclosed in the inter-microbial plaque matrix. Gentle scrubbing will produce adequate cleansing, even in areas of recession or dentinal sensitivity. It can also be used in situations where the bristles of the toothbrush could become irritating creating an "avoiding" reaction.

The forced lockdown of the Covid-19 pandemic brought on other important lessons in hygiene. Parents taught their children to wash their hands often and instructed them not to touch their eyes, nose and mouth with unwashed hands, simple but powerful weapons in helping to prevent disease. As clinicians the treatment of patients is our core mission, but prevention and education are just as important, and will follow a patient for a lifetime. Citing Elisabeth Stone's quote about motherhood: "Making the decision to have a child - it is momentous. It is to decide forever to have your heart go walking around outside your body".

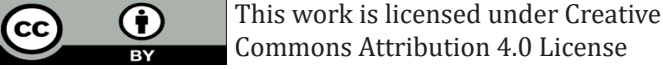

To Submit Your Article Click Here: Submit Article

DOI: $10.32474 /$ IPDOAJ.2020.05.000205

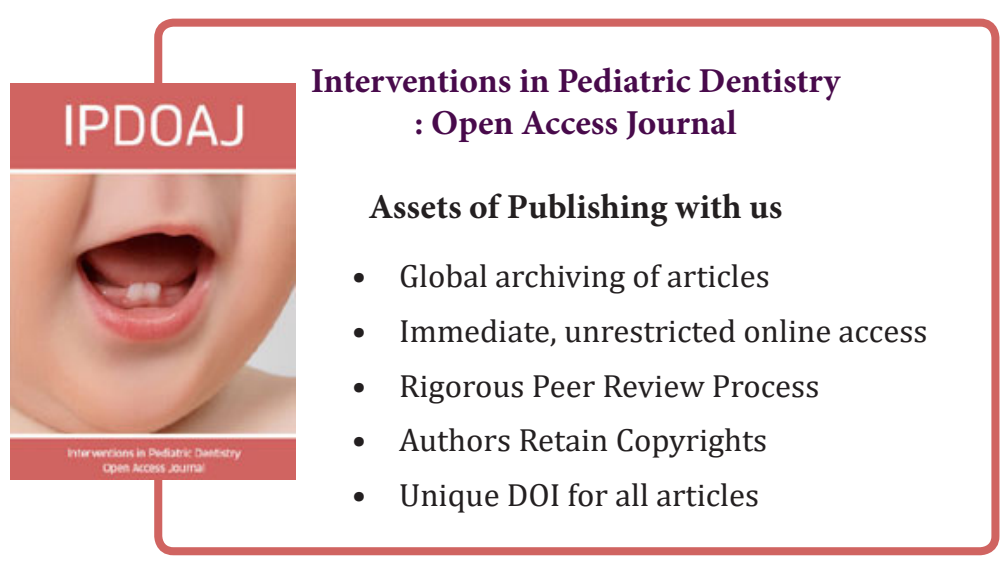

